\title{
Use of recombinant luteinizing hormone for controlled ovarian hyperstimulation in infertile patients
}

\author{
Mônica C. S. Maia ${ }^{1}$, Mário S. Approbato ${ }^{1}$, Tatiana M.da Silva ${ }^{1}$, Eliamar A. B. Fleury ${ }^{1}$, Eliane G. M. Sanchez ${ }^{1}$, \\ Reinaldo S. A. Sasaki ${ }^{1}$ \\ ${ }^{1}$ Laboratory of Human Reproduction of Hospital das Clínicas - Federal University of the State of Goiás (UFG) - \\ Goiânia (GO) - Brazil
}

\begin{abstract}
Controlled ovarian stimulation has become an integral part of a high complexity infertility treatment. Treatment options with recombinant gonadotrophins add more to knowledge on folliculogenesis and ovarian steroidogenesis. Therefore, a literature search was conducted in the following data bases: Medline, Scielo and PubMed. The descriptors/key words used were ovarian stimulation, in vitro fertilization, recombinant luteinizing hormone, supplementation LH. The aim of this study was to review the available literature and to assess the benefits of using recombinant luteinizing hormone associated with recombinant follicle stimulating hormone in different populations who have undergone assisted reproduction procedures.
\end{abstract}

Keywords: ovarian stimulation, in vitro fertilization, supplementation LH

\section{INTRODUCTION}

Reproduction treatment for infertile couples has evolved rapidly since the birth of Louise Brown, the first baby born from in vitro fertilization (IVF) (Steptoe \& Edwards, 1978).

To choose the ovarian stimulation scheme that produces the best follicular response in the most physiological way possible is a very important step in this process. The ovarian stimulation regimens have been enhanced through the use of new therapeutic options (Macklon et al., 2006).

The recombinant luteinizing hormone $(r L H)$ has become available by recombinant DNA technology and is now a new option in ovarian stimulation treatment protocols. It enables us to develop new strategies of ovulation induction, in order to achieve a better control of folliculogenesis. The use of rLH supplementation in IVF is controversial in patients undergoing ovarian stimulation and has been widely debated (Ramachandra et al., 2014; Hill et al., 2012).

Since 1993, rLH has been available for clinical trials and, more recently (2001), it has been registered for therapeutic use. Pharmacokinetic studies with rhLH were undertaken to compare its actions to pituitary and urinary $\mathrm{LH}$. The mean concentration time curve, clearance, and volume distribution at steady states were similar for the three sources of human LH. The distribution half-lives were approximately 0.7 hours, and the terminal half-lives were approximately 10 hours (Porchet et al., 1995).

Specific populations that have been suggested to benefit include patients older than 35 years of age (Bosch et al., 2011), suboptimal responders to ovarian stimulation (Mochtar et al., 2007) and those undergoing a specific GnRH antagonist cycle (Baruffi et al., 2007) or GnRH agonist cycle (Franco et al., 2009).

The aim of this study was to review the available literature and to assess the benefits of using rLH associated with recombinant follicle stimulating hormone ( $\mathrm{rFSH}$ ) in different populations who have undergone assisted reproduction procedures, and the results in number of oocytes retrieved, implantation rate, live birth rate and pregnancy rate.

\section{MATERIALS AND METHODS}

The papers retrieved were those published in the Medline, Scielo and PubMed data bases between the years of 1978 and 2015. The descriptors/key words used were ovarian stimulation, in vitro fertilization and LH supplementation. The words were searched both in Portuguese and English.

The inclusion criteria were: papers published in Portuguese or English between the years of 1978 and 2015; papers with key words pre-established in the titles and/or abstract; studies carried out with human beings. The exclusion criteria were: papers that did not have the pre-established key words in the title and/or abstract. The papers were preselected after the reading of the titles and abstracts. After the papers that fit the criteria were spotted, they were read thoroughly.

\section{RESULTS \\ Recombinant LH administration in patients with poor ovarian response}

The definition of poor ovarian response (POR) in a simple and reproducible manner: it usually indicates a reduction in follicular response, resulting in a reduced number of retrieved oocytes (Ferraretti et al., 2011).

After the introduction of ovarian hyperstimulation in IVF, it soon became clear that ovarian response differs between women. Already in 1983 , the first study that described women with poor response was published (Garcia et al., 1983). Poor response is often related to women with advanced age, in whom the low response to gonadotrophins reflects a physiologic decline in ovarian reserve of primordial follicles (Lawson et al., 2003).

A pathologic decline in number and quality of primordial follicles may also occur in young women. Poor reserve is a common clinical problem, with up to $26 \%$ of IVF cycles resulting in poor response (Keay et al., 1997). In the future this percentage is likely to increase as women continue to postpone childbearing. The diagnosis of poor ovarian reserve is based upon the ovarian response in an IVF treatment cycle and/or patient characteristics such as age, basal FSH, anti-Müllerian hormone (AMH) and/or basal antral follicle count (Sallam et al., 2005).

In women with poor ovarian reserve, the number of mature follicles that develop during stimulation is frequently considered to be insufficient for a successful treatment, leading to cycle cancellation. A Cochrane review suggested that the addition of rLH to controlled ovarian hyperstimulation $(\mathrm{COH})$ with rFSH increases ongoing pregnancy rates in women with poor ovarian reserve [odd ratio (OR) 1.85, 95\% confidence interval (CI) 1.10-3.11] (Mochtar et al., 2007).

Before considering the addition of rLH to IVF stimulation for women with poor ovarian reserve, its costs should be balanced against its potential benefits it will bring in terms of pregnancy (Musters et al., 2012).

Three databases (Medline, Embase and Central) were 
searched (from 1990 to 2011) and included 6433 patients aged $18-45$ years. The co-primary endpoints were number of oocytes retrieved and clinical pregnancy rates. Analyses were carried out for the overall population and patients with poor ovarian response. No significant difference in the number of oocytes retrieved was found between the rFSH plus rLH and rFSH alone groups. However, in poor responders, significantly more oocytes were retrieved with rFSH plus rLH. These data suggest that there is a relative increase in the clinical pregnancy rates of $9 \%$ in the overall population and $30 \%$ in poor responders (Lehert et al., 2014).

\section{Recombinant LH administration in hypogonado- tropic hypogonadism \\ Hypogonadotropic hypogonadism $(\mathrm{HH})$ is a neuro-} endocrine dysfunction presenting with arrested folliculogenesis due to reduced hypothalamic or pituitary activity. In women with $\mathrm{HH}$, successful induction of ovulation can be achieved with pulsatile GnRH therapy, which supplies pulsatile release of gonadotrophins from the pituitary (Filicori et al., 1994).

The exclusive use of FSH in these patients results in fewer developed follicles and lower estradiol levels. In such cases, the addition of LH increases estradiol levels, follicular recruitment and pregnancy rates (European Recombinant LH - Study Group, 1998).

Even though there is no question that rLH administration is necessary in patients with $\mathrm{HH}$, it is still controversial whether its use could enable follicular growth during ovarian stimulation. Although both gonadotropins are necessary for normal follicular development and appropriate steroidogenesis, it has been documented that FSH plays an important role in ovarian stimulation protocols; small amounts of LH are needed to promote a suitable secretion of estradiol and also enable the follicle to undergo final maturation when exposed to hCG (human chorionic gonadotropin). On the other hand, it is known that elevated concentrations of LH in the follicular phase may affect folliculogenesis, resulting in the ovulation of post-mature oocytes (Balasch et al., 1995).

The first randomized clinical study using $\mathrm{rLH}$ in $\mathrm{HH}$ patients (28 with primary and 10 with secondary $\mathrm{HH}$ ) was carried out with 38 patients by administering different doses of rLH (placebo, 25, 75 and 225 IU/ day) in combination with a fixed dose of rFSH (150 IU/ day) (European Recombinant LH Study Group, 1998). Serum estradiol concentrations increased proportionally with increasing rLH and a similar correlation was found in endometrial thickness in rLH up to $75 \mathrm{IU} /$ day dose. Follicular growth was reported in $79 \%$ of patients receiving 75 or $225 \mathrm{IU} /$ day of $\mathrm{rLH}$, while this rate was $27 \%$ in the placebo or $25 \mathrm{IU} /$ day among the rLH receiving group.

This prospective controlled nonrandomized pilot study was designed to investigate whether split daily doses of rLH is more efficacious than the single daily dose in supporting follicular development and ovulation in primary $\mathrm{HH}$. Twenty-seven women with $\mathrm{HH}$ received a 150 IU fixed daily subcutaneous dose of rFSH, supplemented by 75 IU daily dose of rLH administered either as a single dose ( $n=9$; single-dose group) or four equally divided doses ( $n=18$; split-dose group). Women in the split-dose group achieved higher serum estradiol concentrations per follicle, endometrial thickness measurements and numbers of follicles than in the single-dose group (not statistically significant). The OR for ovulation rate was 2.08 (not statistically significant). This study concluded that administering rLH in split daily doses could provide superior results compared to the traditional single daily dose (Awwad et al., 2013).
Recombinant LH administration in older assisted reproduction patients

A possible mechanism behind the beneficial effect of exogenous LH supplementation for women with less sensitive ovaries relates to the decreasing numbers of functional $\mathrm{LH}$ receptors with increasing age. In addition, ovarian androgen secretion, i.e. estrogen precursor secretion capacity, starts to decline as early as before the age of 30 years, again suggesting a diminished capacity of the ovary to respond to LH stimulus with age (Piltonen et al., 2003).

A randomized controlled multicentric study compared rLH supplementation to rFSH in 253 patients aged between 35 and 43 years in a protocol with $\mathrm{GnRH}$ antagonist. Of 253 subjects randomized, 125 received both $\mathrm{rFSH}$ and rLH and 128 received rFSH only. The objectives were to analyze: clinical pregnancy rates, implantation rates, cancellation rates, number of follicles $>15 \mathrm{~mm}$ on the day of hCG administration and number of oocytes obtained. There were no demographic or clinical differences between the groups. They concluded that LH supplementation has no benefit on ongoing pregnancy rates in women of 35 years or older (König et al., 2013).

Vuong et al. (2015), through a randomized controlled trial evaluated a total of 240 women aged $\geq 35$ years undergoing IVF received ovarian stimulation through a $\mathrm{GnRH}$ antagonist protocol. Of the 240 patients randomized to treatment, 120 received $\mathrm{rFSH} / \mathrm{rLH}$ and 120 received $\mathrm{rFSH}$. Their live birth rates, number of oocytes retrieved, implantation rates, miscarriage rates and clinical pregnancy rates did not differ significantly $(P>0.05)$ between $\mathrm{rFSH}+\mathrm{rLH}$ and $\mathrm{rFSH}$.

Bosch et al. (2011) analyzed the impact of LH administration on cycle outcome in ovarian stimulation with $\mathrm{GnRH}$ antagonists. The patients evaluated were under 35 years old $(n=380)$ and those aged 36 to 39 years $(n=340)$. They compared rFSH versus rFSH + rLH administration. Recombinant LH administration significantly increased the implantation rate in patients aged 36 to 39 years. A clinically relevant better ongoing rate per started cycle was found, although the difference was not statistically significant. Patients younger than 36 years did not obtain any benefit from rLH administration.

Recombinant LH administration in normogonadotropic patients

The issue of both LH supplementation and endogenous $\mathrm{LH}$ concentrations in $\mathrm{GnRH}$ antagonist, as well as $\mathrm{GnRH}$ agonist stimulation protocols in normogonadotropic women remains controversial, although a large number of studies on this issue are available. Kol (2005) suggests that the need for exogenous LH could possibly be predicted by the dynamics of endogenous LH levels during stimulation. Rigorous studies with the GnRH agonist GnRH antagonist in multiple dose protocols failed to find an association between endogenous $\mathrm{LH}$ and ongoing pregnancy likelihood (Kolibianakis et al., 2006).

A study evaluated the use of rLH supplementation in an unselected group of IVF patients undergoing follicular stimulation with rFSH after pituitary down-regulation. Group A comprised 122 cycles of rFSH and rLH administered, while group B included 331 cycles using rFSH only, during the same period of treatment. There was no significant difference in any of the endocrine, embryological and outcome parameters measured. The implantation rate of $14.2 \%$ for group A compared with $9.8 \%$ for group B showed a positive trend $(P=0.055)$ (Lisi, 2002).

A total of 244 patients without ovulatory dysfunction, aged $<40$ years and at the first ICSI cycle were divided into two groups matched by age according to an ovarian stimulation scheme: Group I $(\mathrm{n}=122)$ : Down-regulation with $\mathrm{GnRH}$ agonist + rFSH and Group II $(n=122)$ : 
Down-regulation with GnRH agonist + rFSH and rLH (beginning simultaneously). The number of oocytes collected, the number of oocytes in metaphase II, fertilization rates, the mean number of embryos produced per cycle, the mean number of frozen embryos per cycle and cumulative implantation rate were significantly lower in the Group I than in Group II. This study demonstrated the potential benefits with the use of rLH (Franco et al., 2009).

Thus, no consensus has been reached regarding the benefits of adding rLH to this group of patients.

\section{CONCLUSIONS}

There are controversies about the rLH supplementation to FSH during controlled ovarian stimulation. Further comparative studies will be needed to explore and substantiate a proposed beneficial effect of LH supplementation in special patient populations or under certain circumstances, before LH supplementation can routinely be implemented in such situations. It is possible that rLH find its place in ovarian stimulation when nominations are clearly defined and success rates offset the cost of treatment.

\section{CONFLICT OF INTERESTS}

No conflict of interest have been declared.

\section{Corresponding author:}

Mônica Canêdo Silva Maia

Laboratory of Human Reproduction

Hospital Federal University of the State of Goiás (UFG)

Goiânia - GO, Brazil

E-mail: monicalabrep@bol.com.br

\section{REFERENCES}

Awwad JT, Farra C, Mitri F, Abdallah MA, Jaoudeh MA, Ghazeeri G. Split daily recombinant human LH dose in hypogonadotrophic hypogonadism: a nonrandomized controlled pilot study. Reprod Biomed Online. 2013;26:88-92.

Balasch J, Miró F, Burzaco I, Casamitjana R, Civico S, Ballescá JL, Puerto B, Vanrell JA. The role of luteinizing hormone in human follicle development and oocyte fertility: evidence from in-vitro fertilization in a woman with long-standing hypogonadotrophic hypogonadism and using recombinant human follicle stimulating hormone. Hum Reprod. 1995;10:1678-83.

Baruffi RLR, Mauri AL, Petersen CG, Felipe V, Martins AMC, Cornicelli J, Cavagna M, Oliveira JB, Franco JG Jr. Recombinant LH supplementation to recombinant FSH during induced ovarian stimulation in the $\mathrm{GnRH}$-antagonist protocol: a meta-analysis. Reprod Biomed Online. 2007;14:14-25.

Bosch E, Labarta E, Crespo J, Simón C, Remohí J, Pellicer A. Impact of luteinizing hormone administration on gonadotropin-releasing hormone antagonist cycles: an age-adjusted analysis. Fertil Steril. 2011;95:1031-6.

European Recombinant LH Study Group. Recombinant human luteinizing hormone (LH) to support recombinant human follicle-stimulating hormone (FSH)-induced follicular development in LH- and FSH-deficient anovulatory women: A dose- finding study. The European Recombinant LH Study Group. J Clin Endocrinol Metabol. 1998;83:1507-14.

Ferraretti AP, La Marca A, Fauser BC, Tarlatzis B, Nargund G, Gianaroli L. ESHRE consensus on the definition of 'poor response' to ovarian stimulation for in vitro fertilization: the Bologna criteria. Hum Reprod. 2011;26:1616-24.

Filicori M, Flamigni C, Dellai P, Coqniqni G, Michelacci L,
Arnone R. Treatment of anovulation with pulsatile gonadotropin-releasing hormone: prognostic factors and clinical results in 600 cycles. J Clin Endocrinol Metabol. 1994;79:1215-20.

Franco JG, Baruffi RL, Oliveira JB, Mauri AL, Petersen CG, Contart P, Felipe V. Effects of recombinant LH supplementation to recombinant FSH during induced ovarian stimulation in the $\mathrm{GnRH}$-agonist protocol: a matched case-control study. Reprod Biol Endocrinol. 2009;7:58.

Garcia JE, Jones GS, Acosta AA, Wright G Jr. Human menopausal gonadotropin/human chorionic gonadotropin follicular maturation for oocyte aspiration: phase II. Fertil Steril. $1983 ; 39: 174-9$.

Hill MJ, Levens ED, Levy G, Ryan ME, Csokmay JM, DeCherney $\mathrm{AH}$, Whitcomb BW. The use of recombinant luteinizing hormone in patients undergoing assisted reproductive techniques with advanced reproductive age: a systematic review and meta-analysis. Fertil Steril. 2012;97:1108-14.

Keay SD, Liversedge NH, Mathur RS, Jenkins JM. Assisted conception following poor ovarian response to gonadotrophin stimulation. Br J Obstet Gynaecol.1997;104:521-7.

Kol S. To add or not to add LH: consideration of LH concentration changes in individual patients. Reprod Biomed Online. 2005;11:664-6.

Kolibianakis EM, Collins J, Tarlatzis B, Papanikolaou E, Devroey $P$. Are endogenous $\mathrm{LH}$ levels during ovarian stimulation for IVF using $\mathrm{GnRH}$ analogues associated with the probability of ongoing pregnancy? A systematic review. Hum Reprod Update. 2006; 12:3-12.

König TE, van der Houwen LE, Overbeek A, Hendriks ML, Beutler-Beemsterboer SN, Kuchenbecker WK, Renckens CN, Bernardus RE, Schats R, Homburg R, Hompes PG, Lambalk CB. Recombinant LH supplementation to a standard GnRH antagonist protocol in women of 35 years or older undergoing IVF/ICSI: a randomized controlled multicentre study. Hum Reprod. 2013;28:2804-12.

Lawson R, El Toukhy, Kassab A, Taylor A, Braude P, Parsons J, Seed P. Poor response to ovulation induction is a stronger predictor of early menopause than elevated basal FSH: a life table analysis. Hum Reprod. 2003;18:527-33.

Lehert P, Kolibianakis EM, Venetis CA, Schertz J, Saunders $\mathrm{H}$, Arriagada $\mathrm{P}$, Copt S, Tarlatzis B. Recombinant human follicle-stimulating hormone ( $\mathrm{r}-\mathrm{hFSH})$ plus recombinant luteinizing hormone versus r-hFSH alone for ovarian stimulation during assisted reproductive technology: systematic review and meta-analysis. Reprod Biol Endocrinol. 2014; $12: 17$.

Lisi F, Rinaldi L, Fishel S, Lisi R, Pepe GP, Picconeri MG, Campbell A. Use of recombinant $\mathrm{LH}$ in a group of unselected IVF patients. . Reprod Biomed Online. 2002;5:104-8.

Macklon NS, Stouffer RL, Giudice LC, Fauser BC. The science behind 25 years of ovarian stimulation for in vitro fertilization. Endocr Rev. 2006;27:170-207.

Mochtar MH, Van der Veen F, Ziech M, van Wely M. Recombinant luteinizing hormone (rLH) for controlled ovarian hyperstimulation in assisted reproductive cycles. Cochrane Database Syst Rev. 2007;18:CD005070.

Musters AM, van Wely M, Mastenbroek S, Kaaijk EM, Rep- 
ping S, van der Veen F, Mochtar MH. The effect of recombinant LH on embryo quality: a randomized controlled trial in women with poor ovarian reserve. Hum Reprod. 2012;27:244-50.

Piltonen T, Koivunen R, Ruokonen A, Tapanainen JS. Ovarian age-related responsiveness to human chorionic gonadotropin. J Clin Endocrinol Metabol. 2003;88:3327-32.

Porchet HC, Le Cotonnec JY, Neuteboom B, Canali S, Zanolo $\mathrm{G}$. Pharmacokinetics of recombinant human luteinizing hormone after intravenous, intramuscular, and subcutaneous administration in monkeys and comparison with intravenous administration of pituitary human luteinizing hormone. J Clin Endocrinol Metab. 1995;80:667-73.

Ramachandra A, Jamdade K, Kumar P, Adiga SK, Bhat R, Ferrao SR. Is there a need for luteinizing hormone (LH) estimation in patients undergoing ovarian stimulation with gonadotropin releasing hormone $(\mathrm{GnRH})$ antagonists and recombinant follicle-stimulating hormone ( $\mathrm{rFSH})$ ? J Clin Diagn Res. 2014;8:90-2.

Sallam HN, Ezzeldin F, Agameya AF, Rahman AF, El-Garem $Y$. Defining poor responders in assisted reproduction. Int J Fertil Womens Med. 2005;50:115-20.

Steptoe PC, Edwards RG. Birth after reimplantation of a human embryo. The Lancet. 1978;312:336.

Vuong TN, Phung HT, Ho MT. Recombinant follicle-stimulating hormone and recombinant luteinizing hormone versus recombinant follicle-stimulating hormone alone during $\mathrm{GnRH}$ antagonist ovarian stimulation in patients aged $\geq 35$ years: a randomized controlled trial. Hum Reprod. 2015;30: 1188-95. 